\title{
INFLUENCE OF LONGITUDINAL EDGE PROFILING IN SANDWICH PANELS ON INTERPRETATION OF EXPERIMENTAL RESULTS
}

\author{
Monika Chuda-Kowalska \\ Institute of Structural Engineering, Poznan University of Technology, Poland \\ monika.chuda-kowalska@put.poznan.pl
}

\begin{abstract}
Panels with a polyurethane foam core and thin metal facings are considered. Behaviour of these structures depends strongly on the shear rigidity. The recommended methods of identification of the Kirchhoff modulus of the core material $G_{C}$ give different results depending on the used sample. The FEM analysis are performed and the effects of longitudinal edge profiling are presented and discussed.
\end{abstract}

\section{Introduction}

The use of sandwich panels in civil engineering offers many advantages as it leads to structures that are lightweight, cost effective and durable. The main function of the facings is to carry bending, while the role of the core is to keep facings together and carry transverse shear loads. Hence, sandwich panels represent an excellent example of the optimum use of dissimilar materials. Therefore a number of papers is devoted to optimisation of sandwich structures $[1,2]$.

The classical approach to the analysis and design of sandwich plates was presented in $[3,4]$. The engineering aspects of structural response and the effects of transversal load and thermal excitations were presented in [5]. More accurate determination of stress and displacement fields requires application of higher-order theories [6-8], which may involve rather sophisticated numerical analyses. As a result, despite the enhanced accuracy of the advanced formulations, they are less attractive for designers and are rarely used.

Therefore, the aim of this study is to develop the classical approach to accurately predict the global response and/or identify material parameters. Particular attention is focused on the influence of special edge profiling of the plate on its behaviour. Many papers have appeared recently, which take up various problems of identification parameters in multilayer structures [9-11]. The shear rigidity of the panel plays a crucial role in the behaviour of sandwich structures [12]. A group of methods used in identification the shear modulus of the core proposed in the code [13] and in the literature [14] is based on the bending tests of panels with measurement of the transverse displacement $w$. Using the Timoshenko theory the con- 
tributions of bending and shear stiffness coefficients are separated and hence the latter one can be easily determined. The author observed that this method can lead to disadvantageous size effects. Therefore, a new method was proposed. In this method a similar bending test is carried out, but instead of transverse displacement, two angles of rotation are measured in the vicinity of a support. The first one is the angle of cross-section rotation, the latter one is the slope of the panel. The shear modulus $G_{C}$ is calculated directly from the difference between these angles. The new method allows one to avoid errors caused by calculation of bending stiffness $B_{S}$ and deflection due to bending $w_{B}$. The advantage of this method increases when modern tiltmeters are used to measure angles of rotation. These sensors can be easily installed, are precise and allow online data acquisition.

To complete the study on identification of shear modulus of the core other tests were also performed, namely a double-lap shear test and a torsion test of cylindrical samples. However, the evaluation of $G_{C}$ from bending tests seems to be worthy. The first reason is that the tests are simple. Moreover, since the coefficient $G_{C}$ is needed for simulations of mechanical behaviour of slabs in a state of bending and shear, determination of $G_{C}$ from tests of slabs in this state of stress seems to be reasonable. The author carried out numerous experiments for various dimensions and types of loading. The results were reported in [15]. The experiments showed that the evaluated value of $G_{C}$ was strongly affected by the assessed bending rigidity of the plate.

\section{Theoretical background}

In the present paper the theoretical model of the three-layer structure with soft core is applied. It is assumed that the materials of steel facings and polyurethane core are isotropic, homogeneous and linearly elastic. The facings are parallel. In the analysed sandwich panels the Young modulus of the core is much smaller compared than the Young modulus of facings. Therefore, normal stress in the foam core is negligible and the shear stresses are constant in transverse direction $\left(E_{C} / E_{F} \approx 2.0 \cdot 10^{-5} \Rightarrow \sigma_{x C} \approx 0, \tau_{x z C}=\tau_{z x C}=\right.$ const. $)$.

a)

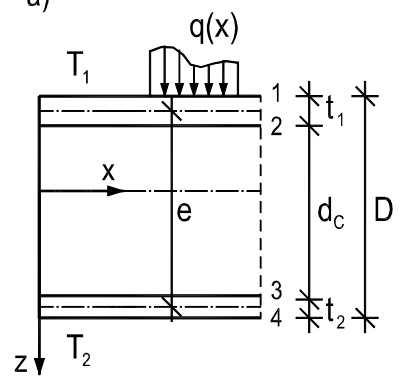

b)

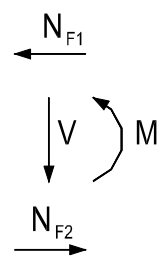

c)

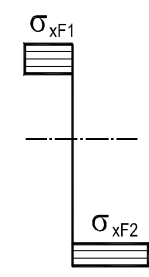

d)

e)

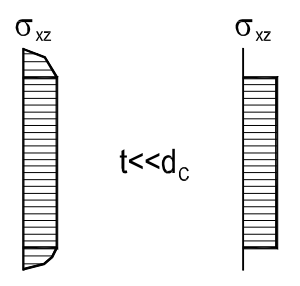

Fig. 1. Distribution of normal and shear stresses in sandwich structures with soft core and thin, flat faces 
The considerations are limited to small strains and displacements (linear geometric relations). Contribution of the shear stress and strain in the facings to the displacement of the panel is neglected because of small thickness of the facings compared to the core. Hence, the Bernoulli hypothesis can be applied independently for both facings. The cross sectional equilibrium condition for a sandwich panel can be written in the form of two independent differential equations [4]:

$$
\begin{gathered}
\frac{B_{F 1}+B_{F 2}}{G_{C} A_{C}} \cdot w^{V I}+\frac{B}{B_{S}} \cdot w^{I V}=\frac{q}{B_{S}}-\frac{q^{\prime \prime}}{G_{C} A_{C}}-\theta^{\prime \prime}, \\
-\frac{B_{F 1}+B_{F 2}}{G_{C} A_{C}} \cdot \gamma^{I V}+\frac{B}{B_{S}} \cdot \gamma^{\prime \prime}=-\frac{q^{\prime}}{G_{C} A_{C}}-\frac{B_{F 1}+B_{F 2}}{G_{C} A_{C}} \cdot \theta^{\prime \prime \prime},
\end{gathered}
$$

where vertical displacement $w$ and shear strain $\gamma$ are the functions of position coordinate $x$. The horizontal displacement $u$ of the mid-plane of the panel is equal to zero. The superscripts of $w$ and $\gamma$ denote the order of the derivative with respect to $x . G_{C}$ and $A_{C}$ denote the shear modulus and cross-sectional area of the core, $q$ is the distributed transverse load and $\theta$ is the initial curvature induced by temperature difference $\Delta T=T_{2}-T_{1}$. In Equations (1) and (2) the normal force is omitted.

Because the bending stiffness of the core is negligible, the total bending stiffness of panel $B$ consists of three parts:

$$
B=B_{S}+B_{F 1}+B_{F 2}
$$

Term $B_{S}$ represents the bending stiffness of the facings with respect to the global centre line of the sandwich panel, whereas $B_{F 1}$ and $B_{F 2}$ are the bending stiffness of the upper and lower facings with respect to their own centre lines.

If the faces of a sandwich panel are thin and flat or they are lightly profiled, the bending stiffness of the faces is small and has a negligible effect on the stress distributions and deflections. In this case it is assumed that $B_{F 1}=B_{F 2}=0$ and $B=B_{S}$. Taking into account the assumptions concerning sandwich element deformation $[12,13]$, the bending moment and shear force can be calculated using:

$$
\begin{gathered}
M=M_{S}=B_{S}\left(\gamma^{\prime}-w^{\prime \prime}-\theta\right), \\
V=V_{S}=G_{C} A_{C} \gamma,
\end{gathered}
$$

where $M_{S}$ and $V_{S}$ correspond to the pure "sandwich" effect.

According to [13], the bending stresses in the flat faces are calculated using:

$$
\sigma_{F 1}=\sigma_{F 1}^{M S}=-\frac{M_{S}}{e A_{F 1}}
$$




$$
\sigma_{F 2}=\sigma_{F 2}^{M S}=\frac{M_{S}}{e A_{F 2}},
$$

where $e, A_{F 1}$ and $A_{F 2}$ denote the distance between the centroids of the faces, the cross-sectional area of the external (upper) and internal (lower) face, respectively. The shear stress in the core is calculated according to:

$$
\tau_{C}=\frac{V_{S}}{A_{C}} .
$$

\section{Formulation of the problem}

The purpose of this paper is the evaluation of influence of longitudinal edge profiling and stiffness of the facings on interpretation of experimental tests results for the identification of the shear rigidity $G_{C}$ of the core. The results obtained from a number of experiments on slabs in a three point bending test are compared with results of numerical simulation. The response of the slabs in experiments is analysed using Timoshenko theory. In numerical simulations FEM is used with 2D and $3 \mathrm{D}$ elements. The slabs with and without edge profiling are examined.

\section{Experimental test}

The code EN 14509 recommends different loading systems shown in Figure 2. The Author used the loading system, which is presented in Figure 2b. The loads are step-wise increased and the total deflection $w$ in the middle point of the span is recorded till the failure occurs. It is assumed, that the deflection can be decomposed on two parts according to:

$$
w=w_{B}+w_{S},
$$

in such a way, that $w_{B}$ and $w_{S}$ represent the deflections due to bending and shear, respectively. The linear part of the function $F\left(w_{S}\right)$ was used for the assessment of the modulus $G_{C}$. According to assumptions presented in 1, the following formulae follow from the Timoshenko theory generalized to sandwich beams:

$$
\begin{gathered}
\Delta w_{B}=\frac{\Delta F \cdot L^{3}}{48 \cdot B_{S}}, \\
B_{S}=\frac{E_{F 1} \cdot A_{F 1} \cdot E_{F 2} \cdot A_{F 2}}{E_{F 1} \cdot A_{F 1}+E_{F 2} \cdot A_{F 2}} \cdot e^{2},
\end{gathered}
$$




$$
\begin{gathered}
G_{C}=\frac{\Delta F \cdot L}{4 \cdot b \cdot d_{C} \cdot \Delta w_{S}}, \\
d_{C}=D-\left(t_{1}+t_{2}\right) .
\end{gathered}
$$

where: $B_{S}$ - the flexural rigidity, $A_{F 1}, A_{F 2}$ - the area of facings, $E_{F 1}, E_{F 2}$ - the Young modulus of facings, $e$ - the distance between centres of facings, $d_{C}$ - the depth of the core and $b$ - the width of the specimen.

a)

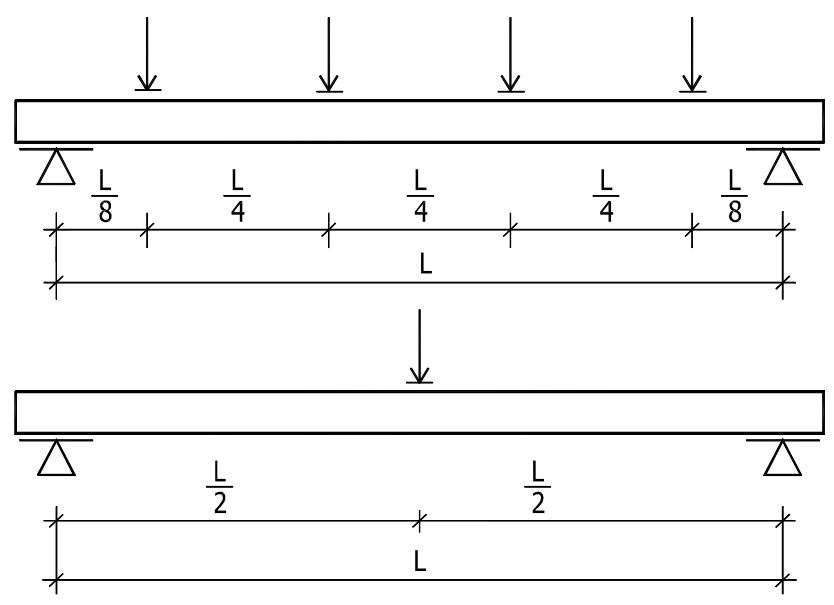

c)

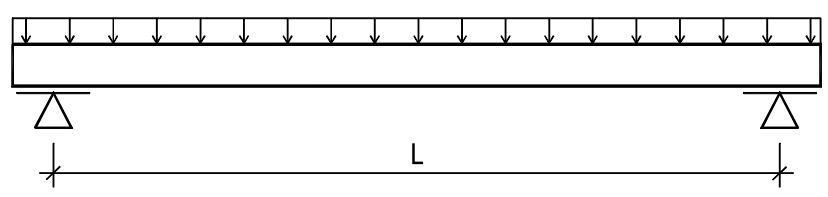

Fig. 2. Scheme of loading system used in bending test [13]

The tests were carried out on a series of sandwich panels with flat facings and a polyurethane core, in which the span $L$ is relatively large. The mean value dimensional parameters are: the total depth of a panel $D=98.5 \mathrm{~mm}$, width $L=4.9 \mathrm{~m}$, facing's thickness $t=0.47 \mathrm{~mm}$ (the steel plate + the zinc coatings). It should be emphasized that in part of tests longitudinal edge profiling of facings was cut off. This operation was necessary in order to estimate the effect of edge stiffening on sandwich behaviour.

The global response of the panel was monitored by continuous measurement of load and displacement. Next, the shear modulus of the core $G_{C}$ was evaluated following the recommendations in the code [13]. The obtained results are presented in Table 1. The discrepancy in $G_{C}$ is evident. 
Experimental results

\begin{tabular}{|c|c|c|}
\hline Type of plates & Total displacement $w[\mathrm{~mm}]$ & $G_{C}[\mathrm{MPa}]$ \\
\hline Edge profiling cut off & 8.90 & 3.78 \\
\hline With edge profiling & 7.50 & 4.74 \\
\hline
\end{tabular}

\section{Numerical model}

The numerical examples refer to structures investigated in real scale during laboratory experiments. Numerical models were prepared in Abaqus system environment. In this paper the author analysed the elastic behaviour of the sandwich panel loaded by the one-line force (Fig. 3).

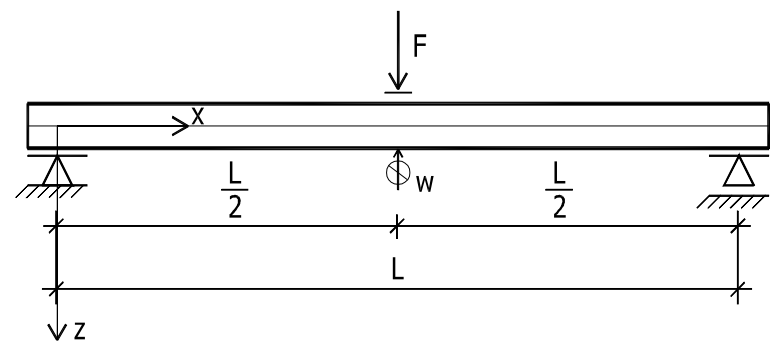

Fig. 3. Used loading system

Two cases were studied. The first one was a plate where longitudinal edge profiling was cut off. In this case the width of the panel was $B=1.0 \mathrm{~m}$. The second one was a panel with edge profiling and $B=1.09 \mathrm{~m}$. In both cases the facings were modelled as a skin element using four node, doubly curved, thin or thick shell, reduced integration, hourglass control, finite membrane strain elements S4R. Following the experiments geometrical and material parameters of the facings were: thickness $t_{F}=0.47 \mathrm{~mm}$, Young modulus $E_{F}=195 \mathrm{GPa}$ and Poisson ratio $v_{F}=0.3$. The value $195 \mathrm{GPa}$ represents a homogenised modulus of the facing composed steel and zinc layers. The core was modelled using eight node linear brick elements C3D8R. Its parameters were: thickness $d_{C}=97.56 \mathrm{~mm}$, Poisson ratio $v_{C}=0.05$ and shear modulus as the value obtained in the respective, real experiment. The edge profiling was modelled as stringers using two node linear beam elements B31. These elements have the same material parameters as the facings. The shape and size of these elements mapped real edge profiling and showed in Figure 4. The panel is supported by two basing plates, which are modelled as rigid bodies. For the left supporting base plate all three translations and the rotations with respect to axes $x$ and $z$ are equal to zero. Unconstrained rotation with respect to the axis $y$ is assumed. The right base plate has free translation in the direction $x$ 
(Fig. 3). Important point of the model is a connection between facings - core and facings - edge profiling. Abaqus provides a very useful tool to model shell and beam elements as a part of 3D brick elements, i.e. shell elements are created directly on the brick element nodes as well as beam elements which are built on the edge nodes of the brick elements. Therefore, no interface interaction e.g. contact or tie connection need to be modelled, which simplifies the problem and drastically reduces the computational time without losing the objectivity of results.
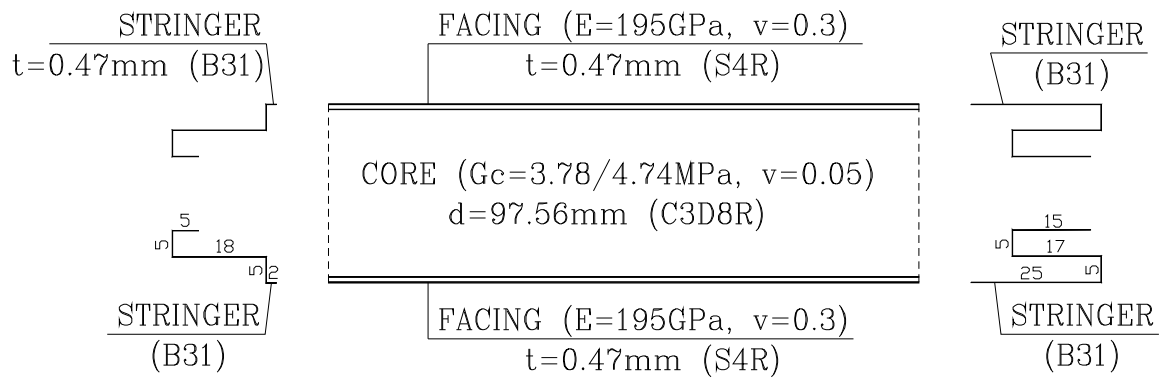

Fig. 4. The shape of edge profiling

\section{Comparison of the experimental and numerical results}

The numerical model of the panel with flat facings in the state of bending and shear was verified. Therefore, the shear modulus $G_{C}$ obtained from various kinds of laboratory tests was introduced. The numerical response of the panel was compared to the experimental behaviour.

Table 2

Comparison of results

\begin{tabular}{|c|c|c|}
\hline Type of plates & $G_{C}[\mathrm{MPa}]$ & Total displacement $w[\mathrm{~mm}]$ \\
\hline Experimental model & & \\
A: edge profiling cut off $\quad(\mathrm{B} 1=1.0 \mathrm{~m})$ & 3.78 & 8.89 \\
B: with edge profiling $\quad(\mathrm{B} 2=1.09 \mathrm{~m})$ & 4.74 & 7.54 \\
\hline Numerical model A & $\mathbf{3 . 7 8}$ & $\mathbf{8 . 7 4}$ \\
(edge profiling cut off, B1 =1.0 m) & 4.74 & 8.10 \\
\hline Numerical model B & $\mathbf{3 . 7 8}$ & 7.63 \\
(with edge profiling, B2 $=1.09 \mathrm{~m})$ & 4.74 & 7.11 \\
\hline
\end{tabular}

Numerical model A shows good agreement with real behaviour when we introduce the shear modulus identified on samples without edge profiling $\left(G_{C}=\right.$ 
$=3.78 \mathrm{MPa}$ ). We can observe similar behaviour in model B. It means that modulus $G_{C}$ should be identify on samples without edge profiling.

\section{Conclusions}

The core flexibility plays a crucial role in assessment of the functional response of sandwich panels, such as load-bearing capacity and deflection and hence maximum permissible span. The bending test on sandwich plate with longitudinal edge profiling allows to observe its behaviour in real scale. This kind of test is allowed by the code [13] also for the identification of shear modulus $G_{C}$ of the core material. In this study it was demonstrated by the way of a number of experiments and computer simulations that the value of $G_{C}$ is very sensitive to assumed bending stiffness of the panel. Neglecting the contribution of the edge profiling to the total bending stiffness of the plate can lead to $25 \%$ overestimation of $G_{C}$.

\section{References}

[1] Icardi U., Ferrero L., Optimisation of sandwich panels with functionally graded core and faces, Composite Science and Technology 2009, 69, 575-585.

[2] Studziński R., Pozorski Z., Garstecki A., Optimal design of sandwich panels with soft core, Journal of Theoretical and Applied Mechanics 2009, 47(3), 685-699.

[3] Allen H.G., Analysis and Design of Structural Sandwich Panelsn Pergamon Press, London 1969.

[4] Stamm K. et al., Sandwichkonstruktionen: Berechnung, Fertigung, Ausführung, Springer-Verlag, Wien 1974.

[5] Błaszczuk J., Pozorski Z., Engineering aspects of structural response of multi-span sandwich panels, Scientific Research of the Institute of Mathematics and Computer Science 2010, 2(9), 5-15.

[6] Bickford W.B., Consistent higher order beam theory, Developments in Theoretical and Applied Mechanics 1982, 11, 137-150.

[7] Levinson M., An accurate, simple theory of the statics and dynamics of elastic plates, Mechanics Research Communications 1980, 7, 343-350.

[8] Reddy J.N., A simple higher-order theory for laminated composite plates, Journal of Applied Mechanics 1984, 51, 745-752.

[9] Harders H. et al., Influence of cell wall shape and density on the mechanical behaviour of 2D foam structures, Acta Materialia 2004, 53, 1335-1345.

[10] Mills N.J., Polymer Foams Handbook. Engineering and Biomechanics Applications and Design Guide, Butterworth - Heinemann 2007.

[11] Ramsteiner F. et al., Testing the deformation behaviour of polymer foams, Polymer Testing 2001, 20, 661-670.

[12] Błaszczuk J., Pozorski Z., The analysis of the influence of core compression effect on the determination of the shear modulus of the sandwich panel core, Scientific Research of the Institute of Mathematics and Computer Science 2012, 2(11), 5-13.

[13] EN 14509 Self-supporting double skin metal faced insulating panels - Factory made products Specifications. Warsaw 2007. 
[14] Juntikka R., Hallstorm S., Shear characterization of sandwich core materials using four-point bending, Journal of Sandwich Structures \& Materials 2007, 9, 1 67-94.

[15] Chuda-Kowalska M. et al., Experimental determination of shear rigidity of sandwich panels with soft core, Proc. of 10th International Conference Modern Buildings Materials, Structures and Techniques, Vol. I, ed. P. Vainiûnas, E.K. Zavadskas, Vilnius, Lithuania, VGTU 2010, 56-63. 
